\title{
Abnormal expression of TIP30 and arrested nucleocytoplasmic transport within oligodendrocyte precursor cells in multiple sclerosis
}

\author{
Jin Nakahara, ${ }^{1,2}$ Kohsuke Kanekura, ${ }^{2}$ Mikiro Nawa, ${ }^{2}$ Sadakazu Aiso, ${ }^{2}$ and Norihiro Suzuki1 \\ 1Department of Neurology and 2Department of Anatomy, Keio University School of Medicine, Tokyo, Japan.
}

\begin{abstract}
Oligodendrocyte precursor cells (OPCs) persist near the demyelinated axons arising in MS but inefficiently differentiate into oligodendrocytes and remyelinate these axons. The pathogenesis of differentiation failure remains elusive. We initially hypothesized that injured axons fail to present Contactin, a positive ligand for the oligodendroglial Notch1 receptor to induce myelination, and thus tracked axoglial Contactin/Notch1 signaling in situ, using immunohistochemistry in brain tissue from MS patients containing chronic demyelinated lesions. Instead, we found that Contactin was saturated on demyelinated axons, Notch1-positive OPCs accumulated in Contactin-positive lesions, and the receptor was engaged, as demonstrated by cleavage to Notch1intracellular domain (NICD). However, nuclear translocalization of NICD, required for myelinogenesis, was virtually absent in these cells. NICD and related proteins carrying nuclear localization signals were associated with the nuclear transporter Importin but were trapped in the cytoplasm. Abnormal expression of TIP30, a direct inhibitor of Importin, was observed in these OPCs. Overexpression of TIP30 in a rat OPC cell line resulted in cytoplasmic entrapment of NICD and arrest of differentiation upon stimulation with ContactinFc. Our results suggest that extracellular inhibitory factors as well as an intrinsic nucleocytoplasmic transport blockade within OPCs may be involved in the pathogenesis of remyelination failure in MS.
\end{abstract}

\section{Introduction}

Natural remyelination of the central nervous system often occurs spontaneously in various demyelinating diseases, including MS, but only in a subset of patients $(1,2)$. Oligodendrocyte precursor cells (OPCs) are typically preserved in or around demyelinated lesions of MS $(3,4)$; nonetheless, persistent demyelination even in the absence of active inflammation is a hallmark of chronic MS and leads to sustained neurological deficits in patients. Elucidating the pathogenesis of this failure is essential in order to identify therapeutic targets for the induction of remyelination in MS.

It remains elusive why preserved OPCs persist near demyelinated axons but do not differentiate into mature oligodendrocytes and consequently remyelinate axons. Multiple environmental factors have been proposed to contribute to the remyelination failure (5). Assuming that the preserved OPCs are naive and have no intrinsic dysfunction inhibiting their differentiation, remyelination failure may be due to an excess of inhibitory extracellular signals that block differentiation or a deficit in signals promoting differentiation. As for the former possibility, it has been proposed that nearby astrocytes may play a role either by expression of Jagged1, which in turn negatively activates oligodendroglial Notch 1 receptor and promotes the expression of Hes5, a transcription factor inhibiting the differentiation (6), or by production of hyaluronan,

Conflict of interest: The authors have declared that no conflict of interest exists. Nonstandard abbreviations used: ADLD, autosomal dominant leukodystrophy; LFB, luxol fast blue; MAG, myelin-associated glycoprotein; NFR, nuclear fast red; NICD, Notch1-intracellular domain; NLS, nuclear localizing signal(s); NPC, nuclear pore complex; OPC, oligodendrocyte precursor cell; PDI, protein disulfide isomerase; TIP30, TAT-interacting protein $30 \mathrm{kDa}$.

Citation for this article: J. Clin. Invest. 119:169-181 (2009). doi:10.1172/JCI35440. which prevents the maturation process of oligodendrocytes (7). PSA-NCAM abnormally expressed on demyelinated axons (8), LINGO-1 expressed on astrocytes and macrophages $(9,10)$, and myelin debris (11) may also inhibit the differentiation of OPCs.

Regarding the latter possibility, it remains unexamined whether signals promoting oligodendrocyte differentiation are reduced in MS lesions. Recently, axonal Contactin was reported to play a major role in the induction of oligodendroglial differentiation (12). Axonal Contactin, in contrast with astrocytic Jagged1, positively activates oligodendroglial Notch1 receptor and induces oligodendroglial differentiation. However, surface expression of Contactin is dependent on neuronal electrical activity (13), and axonal pathologies are observed in MS lesions $(4,14)$. We therefore initially hypothesized that the upregulation of Contactin may be altered in the preserved axons, possibly due to impaired electrical activity, and that this reduction contributes to the failure of remyelination in MS.

The current study was undertaken to evaluate axoglial Contactin/Notch1 signaling (12) in MS lesions. For this purpose, we tracked the signaling pathway immunohistochemically in situ using 10 autopsied chronic MS brains. In contrast with our initial hypothesis, we found that the expression of Contactin was upregulated on demyelinated axons. Further analysis revealed that Notch1-positive OPCs accumulated in Contactin-positive lesions and that the receptor was engaged, as shown by cleavage to Notch1intracellular domain (NICD); nonetheless, nuclear translocalization of NICD, required for myelinogenesis, was virtually absent in these cells. Nuclear translocalization of NICD is mediated by Importin $\beta$ recognizing nuclear localizing signals (NLS) (15-17). We found that Importin $\beta$ was colocalized with NICD in the OPCs, 


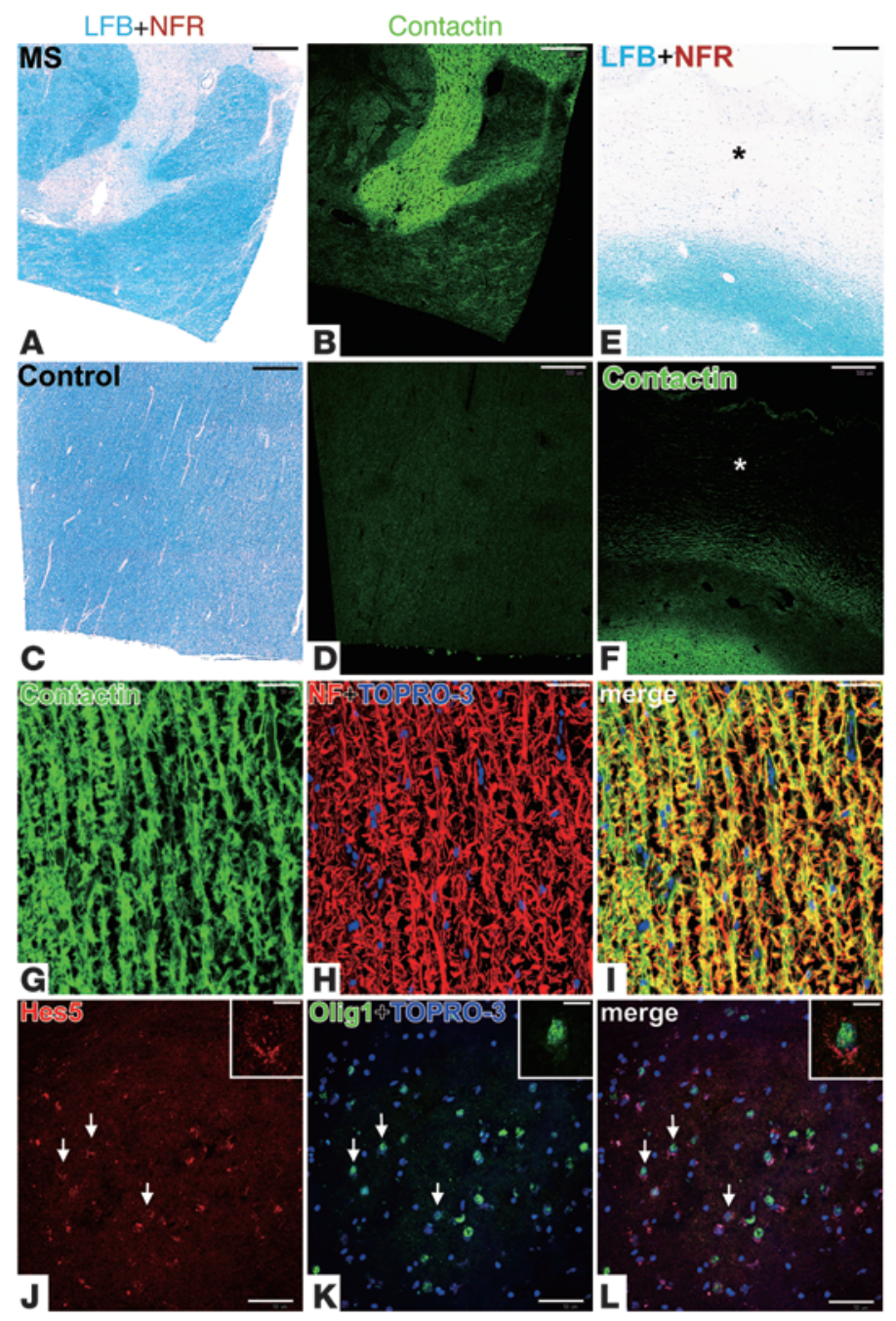

\section{Figure 1}

Remyelination failure in MS may not be due to loss of axonal Contactin stimuli or induction of nuclear Hes5 by astrocytes. (A) LFB staining showing normal-appearing white matter (blue) and a demyelinated lesion (white) in an MS brain (frontal lobe cerebral lesion from 47-year-old female with secondary progressive MS; disease duration: 20 years). Cntn, Contactin. (B) Section consecutive to $\mathbf{A}$, showing that the expression of Contactin is upregulated in the demyelinated lesion. (C) Demyelination is not apparent in a control subject (parietal lobe cerebral specimen from 81-yearold female without brain disease). (D) Section consecutive to C showing that Contactin is expressed at a low degree; thus, the expression is unremarkable in photograph at lower magnification. Overexpression of Contactin in MS demyelinated lesions are observed in all $10 \mathrm{MS}$ specimens included in this study (the other 9 MS samples are shown in Supplemental Figure 1). ( $E$ and F) Contactin immunoreactivity was lost in demyelinated areas where severe tissue damage was evident (asterisks; frontal lobe cerebral lesion from 59-year-old male with primary progressive MS. Isotype controls are shown in Supplemental Figure 2. (G-I) Overexpression of Contactin in MS demyelinated lesions occurs on the surfaces of axons positive for neurofilament (NF). Photographs at lower magnification are shown in Supplemental Figure 3. (J-L) Preserved OPCs in MS lesions are positive for nuclear Olig1 (24) and express Hes-5 in the cytoplasm but not in the nucleus (arrows and insets). Scale bars: $500 \mu \mathrm{m}(\mathbf{A}-\mathbf{F}) ; 50 \mu \mathrm{m}$ (G-L); $10 \mu \mathrm{m}$ (insets in $\mathbf{J}-\mathbf{L}$ ). although TAT-interacting protein $30 \mathrm{kDa}$ (TIP30), a direct inhibitor of Importin $\beta(18,19)$, was upregulated in such cells. Indeed, TIP30-positive cells were significantly increased in chronic demyelinated plaques when compared with shadow plaques where remyelination had taken place. We also show that overexpression of TIP30 in OPC cell lines in vitro resulted in cytoplasmic entrapment of NICD and arrest of differentiation upon stimulation with Contactin-Fc. Together, our observations suggest that not only extracellular inhibitory factors but also an intrinsic nucleocytoplasmic transport blockade within OPCs may be involved in the pathogenesis of remyelination failure in MS.

\section{Results}

Demyelinated axons in MS express Contactin. Using autopsied brain samples from 10 chronic MS patients (including 2 relapsing/ remitting MS, 2 primary progressive MS, 5 secondary progressive MS, and 1 without clinical course information available; average age of 54.9 years at the time of autopsy [range: $47-72$ years], with disease durations of 12-29 years; 9 chronic demyelinated [inactive] lesions and 1 actively demyelinating lesion, as listed in our previous report; ref. 20), we detected increased expression of Contactin within demyelinated lesions in all samples examined, regardless of clinical courses or ages (Figure 1, A and B, and Supplemental
Figure 1; supplemental material available online with this article; doi:10.1172/JCI35440DS1), compared with that in 5 normal control subjects (average age of 76.2 years at the time of autopsy [range: 54-91 years], as listed in our previous report; ref. 20; Figure 1, C and $\mathrm{D}$, and data not shown). Contactin immunoreactivity was lost in demyelinated lesions where severe tissue damage was evident (as indicated by a severe reduction in nucleated cells and a sparse axonal architecture; Figure 1, E and F), suggesting that the observations are unlikely to be artifacts due to degenerative processes in the lesions (isotype controls for immunohistochemistry are shown in Supplemental Figure 2). Contactin was expressed on axonal surfaces (Figure 1, G-I, and Supplemental Figure 3), indicating that the remyelination failure was unlikely to be due to the lack of axonal stimuli, at least in the context of axoglial Contactin/Notch1 signaling for myelinogenesis (12). Interestingly, Contactin immunoreactivity appeared grossly saturated and was detected on axonal surfaces as well as at intercellular matrix in some MS lesions (e.g., Figure 1B), while it was more faintly stained and localized primarily to axonal surfaces in other lesions (e.g., Supplemental Figure 1R). Although Contactin is a glycosylphosphatidylinositol-anchored neuronal cell adhesion molecule attached to cell membrane, a soluble form of Contactin, which promotes neurite outgrowth, is also produced and becomes detectable in the cerebrospinal fluid during 


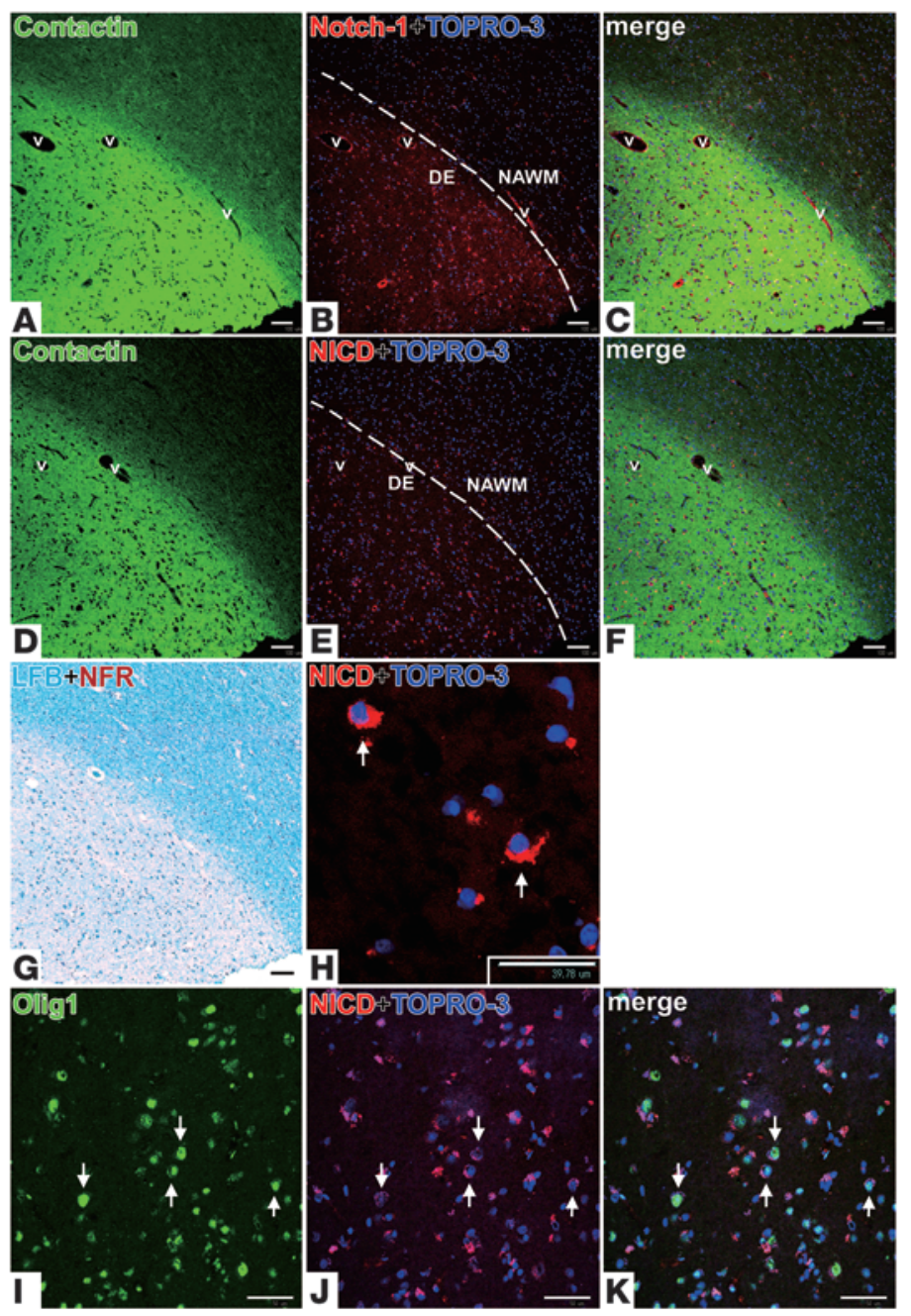

\section{Figure 2}

Accumulations of Notch1-positive OPCs and receptor activation take place; nonetheless, nuclear translocalization of NICD fails in OPCs in MS lesions. (A-C) Notch1-positive cells are increased in a Contactin-positive demyelinated MS lesion in comparison with normal-appearing white matter (border demarcated by broken line). (D-F) Immunostaining in a section consecutive to $\mathbf{A}-\mathbf{C}$ with a specific $A$ b against NICD, which does not recognize full-length Notch1, reveals that the activation of Notch1 and consequent cleavage to NICD took place in these cells. (G) LFB staining in a section consecutive to $\mathbf{D}-\mathbf{F}$ is shown. (H) A photograph at higher magnification showing that NICD, which is expected to translocate into nucleus, is aggregated in cytoplasm but not nucleus (arrows) in the OPCs (see also Supplemental Figure 5 for results from other MS specimens). (I-K) These NICD-positive cells are positive for nuclear Olig1, indicative of oligodendroglial cells recruited for remyelination (24) (arrows). DE, demyelinated lesion; NAWM, normalappearing white matter; v, vessels. Scale bars: $100 \mu \mathrm{m}(\mathbf{A}-\mathbf{G})$; $40 \mu \mathrm{m}(\mathbf{H}) ; 50 \mu \mathrm{m}(\mathbf{I}-\mathbf{K})$.

development (21). Soluble Contactin is also detectable in the cerebrospinal fluid from some but not all MS patients (Jin Nakahara, unpublished observations); thus, the diversity of Contactin immunoreactivity may reflect the availability of the soluble form at the site in addition to the density of residual axons.

The same Notch 1 receptor is shared by 2 different ligands, axonal Contactin and astrocytic Jagged1, although the former promotes (12) whereas the latter inhibits (6) the differentiation of OPCs into mature oligodendrocytes. Therefore, we next hypothesized that despite the increased expression of Contactin on demyelinated axons, Jagged1/Notch1 signaling between astrocytes (and possibly macrophages in acute lesions; ref. 22) and OPCs may competitively dominate and induce the expression of Hes5, a transcription factor that inhibits the differentiation of oligodendrocytes (23). It is noteworthy that the degree to which astrocytes accumulated in MS lesions varied from case to case and that demyelinated lesions were not always occupied by astrocytes (Supplemental Figure 4). In chronic cases in which astrocytes and macrophages are not predominant, axonal Contactin and astrocytic Jagged 1 may not compete for the Notch 1 receptor. Our results showed that Hes5 was detected weakly in OPCs recruited for remyelination (as indicated by nuclear Olig1 transcription factor; ref. 24) in the demyelinated lesions and that the expression was exclusively cytoplasmic (Fig- ure 1, J-L). A few exclusive nuclear Hes5-positive cells found in the lesions were mostly negative for Olig 1 and therefore not OPCs involved in remyelination. Both Hes5 and Olig1 are basic helixloop-helix transcription factors whose primary functions take place in the nucleus; cell fates are regulated by the movement of these factors between nucleus and cytoplasm (25). Nuclear Hes5 is negatively involved (23), whereas nuclear Olig1 is positively involved (24) in the differentiation of OPCs into mature cells; thus, our observations showing nuclear Olig1 but cytoplasmic Hes5 in MS lesions indicate that the remyelination failure is unlikely to be the consequence of astrocytic Jagged1-induced inhibition (6) dominating axonal Contactin-induced differentiation (12).

Detection of axoglial Contactin/Notch1 signaling within OPCs in MS lesions. The above results suggest that the remyelination-promoting pathways downstream of Contactin/Notch1 engagement may be impaired in OPCs. When Notch 1 is activated upon binding of axonal Contactin, sequential proteolytic cleavages take place, resulting in production of NICD. NICD contains internal NLS (15), which direct translocation of the protein into the nucleus for the start of myelinogenesis (12). In order to clarify whether the receptor activation and the cleavage of Notch1 takes place properly in Contactin-positive demyelinated lesions, we examined the lesions with 2 Abs: 1 against full-length Notch1 and 1 that spe- 


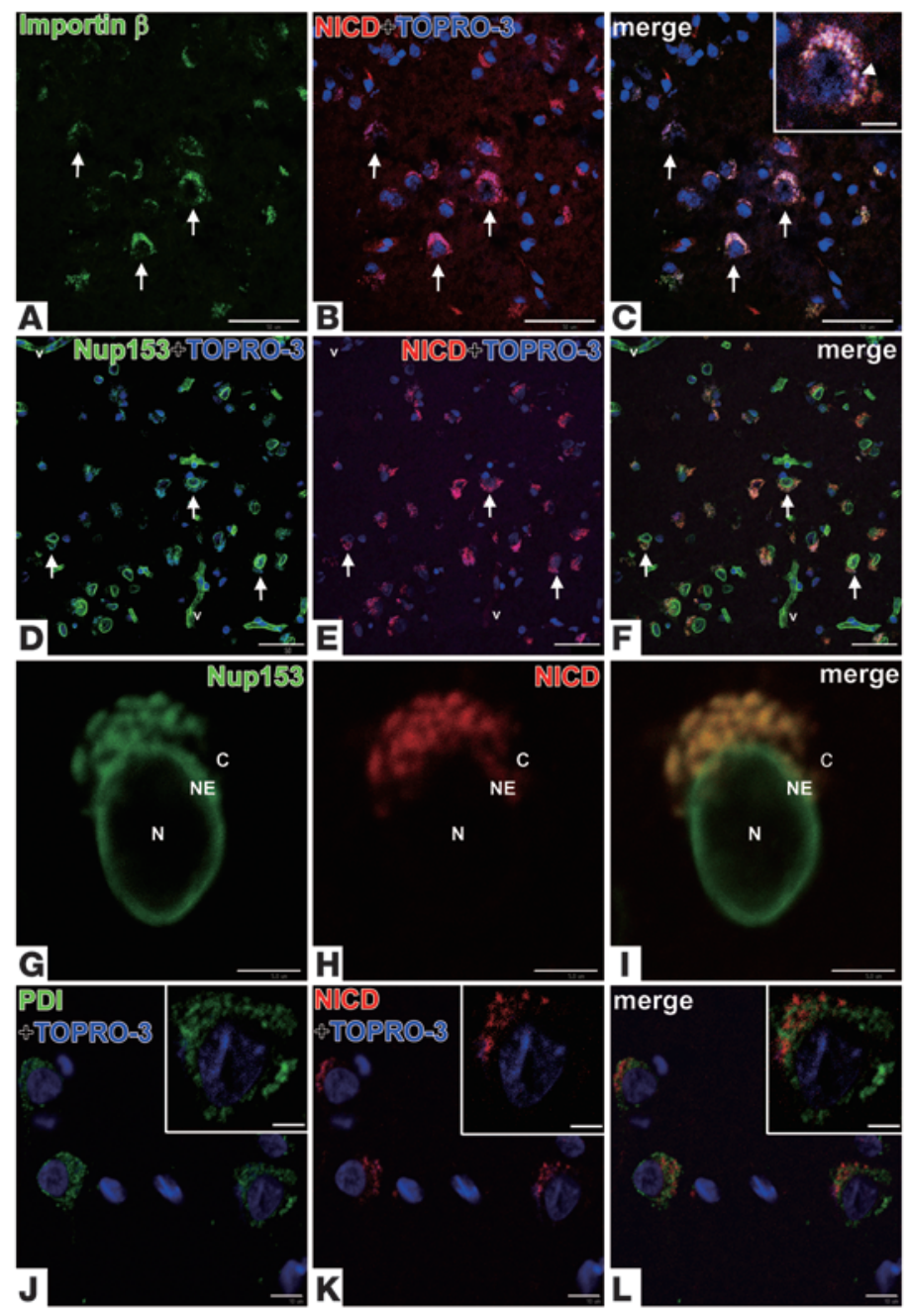

\section{Figure 3}

Nuclear transporter Importin $\beta$ and NPC protein Nup153 are not diminished but are aggregated with NICD in cytoplasm of OPCs in MS lesions. (A-C) The expression of Importin $\beta$, an indispensable nuclear transport protein, is increased in an MS demyelinated lesion and colocalized with the NICD aggregates in the OPCs (arrows and arrowhead in inset). Photographs at lower magnification are shown in Supplemental Figure 6; note that most, if not all, of these cytoplasmic NICD-aggregated cells are positive for Olig1, an OPC marker (24), as shown in Figure 2. (D-F) NPC protein Nup153, required for entrance into the nucleus, is also expressed in the OPCs (arrows). (G-I) Photographs at higher magnification showing that the expression of Nup153 is localized at the nuclear envelope and also colocalized with the NICD-cytoplasmic aggregates. (J-L) NICD aggregates are not localized within ER structures (positive for PDI). N, nucleus; NE, nuclear envelope; C, cytoplasm. Scale bars: $50 \mu \mathrm{m}$ (A-F); $10 \mu \mathrm{m}$ (insets in $\mathbf{C}$; $\mathbf{J}-\mathbf{L}) ; 5$ m (G-I; insets in $\mathbf{J}-\mathbf{L}$ ). cifically detects $\mathrm{Val}_{1744}$-cleaved NICD but not full-length Notch1 or Notch1 cleaved at other positions. As expected, Notch1-positive cells accumulated in Contactin-positive demyelinated lesions (Figure 2, A, B, C, and G). NICD-positive cells were detected in the same manner except for endothelial cells in small vessels, which were positive for Notch 1 but rather weakly positive or negative for NICD (Figure 2, D-G). These results indicate that the receptor engagement and sequential cleavages of Notch1 did indeed take place in these cells. However, NICD was aggregated in the cytoplasm, and nuclear NICD-positive cells were virtually undetected in all cases examined (Figure $2 \mathrm{H}$ and Supplemental Figure 5; note that NICD immunoreactivity was mostly undetected in control subjects, as shown in the latter figure). These cytoplasmic NICDpositive cells were mostly positive for nuclear Olig1 (Figure 2, I-K) and cytoplasmic CNPase (not shown), indicative of OPCs.

It is unlikely that we were observing NICD that had once been imported into nucleus and then subsequently exported to cytoplasm, since a study that followed the activation of Notch 1 in real time showed, using the same Ab, that NICD is rapidly degraded after nuclear translocation and not detected in cytoplasm afterward (26). Our observations thus indicate that the nuclear translocalization of NICD failed to occur and that axoglial Contactin/ Notch1 signaling was consequently distended in these OPCs.
Cytoplasmic aggregations of NICD with Importin $\beta$, Nup153, and Lamin $B 1$. Next, we addressed the question of why nuclear translocalization of NICD is impaired in the OPCs, making them unresponsive to axonal signals for myelination. NICD is a $110-\mathrm{kD}$ a protein containing NLS (15). Molecules smaller than 20-40 kDa may passively diffuse through the nuclear pore complex (NPC) to enter the nucleus, whereas larger molecules, including NICD, require coupling with nuclear transporters (16). Importin $\beta$ is an essential transporter indispensable for nuclear translocation of NLS-tagged proteins such as NICD $(16,17)$ and is a critical determinant of nuclear import velocity (27). A previous microarray analysis detected reduced Importin $\beta$-mRNA levels in MS lesions compared with control brain tissues (28); thus, we hypothesized that a deficiency of Importin $\beta$ may underlie the observed nuclear translocation failure of NICD. This is unlikely, however, since Importin $\beta$ protein was clearly detected in the demyelinated lesions, with more intense signals than in nearby, normal-appearing white matter (Supplemental Figure 6) or in control subjects (not shown). Furthermore, the Importin $\beta$ we detected colocalized with the cytoplasmic NICD aggregates in the OPCs (Figure 3, A-C).

We next considered degradation of the NPC, the entrance to the nucleus, as a possible cause for the nucleocytoplasmic transport failure. Nup153 is a key protein involved in the formation of NPC; it 


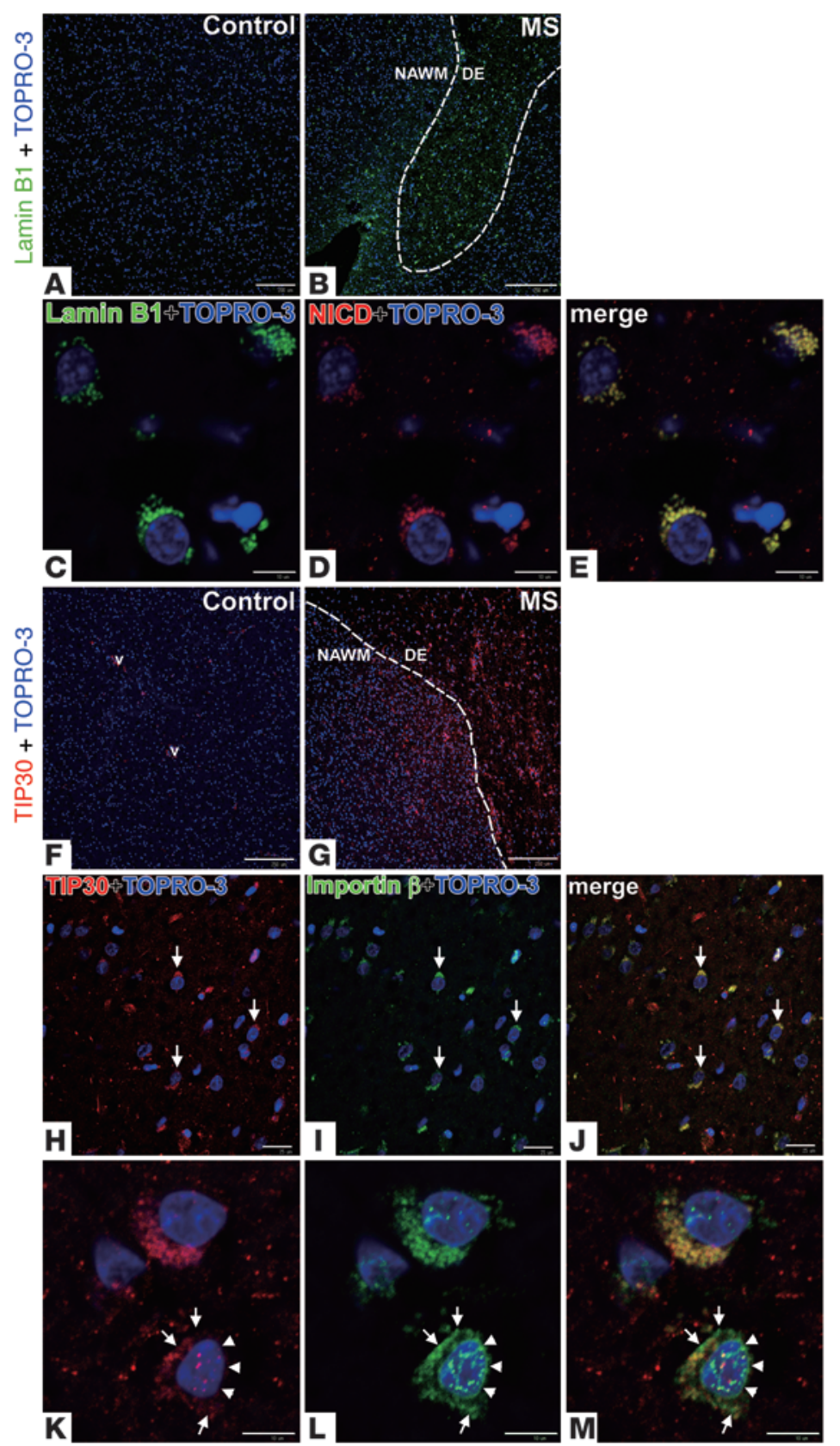

\section{Figure 4}

Abnormal expression of Lamin B1 and TIP30 in OPCs in MS lesions. (A) The expression of Lamin B1 is virtually undetectable in normal cerebral white matter from a control subject. (B) The expression is increased in a MS demyelinated lesion (surrounded by broken line) compared with nearby normal-appearing white matter. (C-E; higher magnification) Overexpressed Lamin B1 colocalizes with cytoplasmic NICD aggregates in the OPCs (note that the most of these cytoplasmic NICD-aggregated cells are positive for Olig1, an OPC marker [ref. 24], as shown in Figure 2), but not with nuclear envelopes. (F) TIP30 is undetectable except in small vessels in normal cerebral white matter from a control subject. (G) TIP30 expression is markedly increased in a MS demyelinated lesion (demyelinated area and normal-appearing white matter demarcated by broken line). $(\mathbf{H}-\mathbf{J})$ Overexpressed TIP30, an inhibitor of Importin $\beta$ (19), is associated with Importin $\beta$ in the OPCs (arrows). ( $\mathbf{K}-\mathbf{M}$; higher magnification) In a few cells with relatively lower expression of TIP30 (e.g., the lower cell in the pictures), Importin $\beta$ that is not colocalized with TIP30 (arrows) gathers near the nuclear envelope (arrowheads); some appears to have entered the nucleus. Scale bars: $250 \mu \mathrm{m}$ (A, B, F, and $\mathbf{G}) ; 10 \mu \mathrm{m}$ (C-E and $\mathbf{K}-\mathbf{M}) ; 25 \mu \mathrm{m}(\mathbf{H}-\mathbf{J})$. associates with and is required for the Importin $\beta$-mediated nuclear imports (29). Certain viruses, such as poliovirus, cause abnormal degradation of Nup153 and consequently inhibit nuclear translocation of NLS-tagged proteins carried by Importin $\beta$ (30). However, Nup153 immunoreactivity of OPCs in MS lesions showed normal-appearing nuclear envelopes, though some Nup153 appeared to be abnormally localized outside the envelopes and within the cytoplasm, associated with the NICD aggregates (Figure 3, D-I). When Nup153 is expressed in excess in vitro, it is known to induce the formation of annulate lamellae, an expansion of the portion of the ER contiguous with the nuclear envelope (31). To exclude the possibility that the observed NICD aggregates in the OPCs are located in annulate lamellae along with Nup153, we examined their subcellular localization using protein disulfide isomerase (PDI) as an ER marker. The results showed that the aggregates do not localize within ER structures (Figure 3, J-L). The NICD aggregates and associated Nup153 are thus cytoplasmic.

Abnormal aggregations of NPC protein Nup153 in cytoplasm are highlighted in the above results. The stability of Nup153 is largely determined by its interactions with intermediate filaments known as lamins (32). Lamins are essential components of the nuclear envelope and include multiple members whose expression levels vary from tissue to tissue. Recently, genetic duplications in the Lamin $\mathrm{B} 1$ gene and consequent overexpression of the protein have been reported as a cause of human adult-onset autosomal dominant leukodystrophy (ADLD) (33), a hereditary demyelinating disease without signs of remyelination. Lamin B1 a Lamin protein that is expressed at a relatively low degree in healthy brains (34), with the 

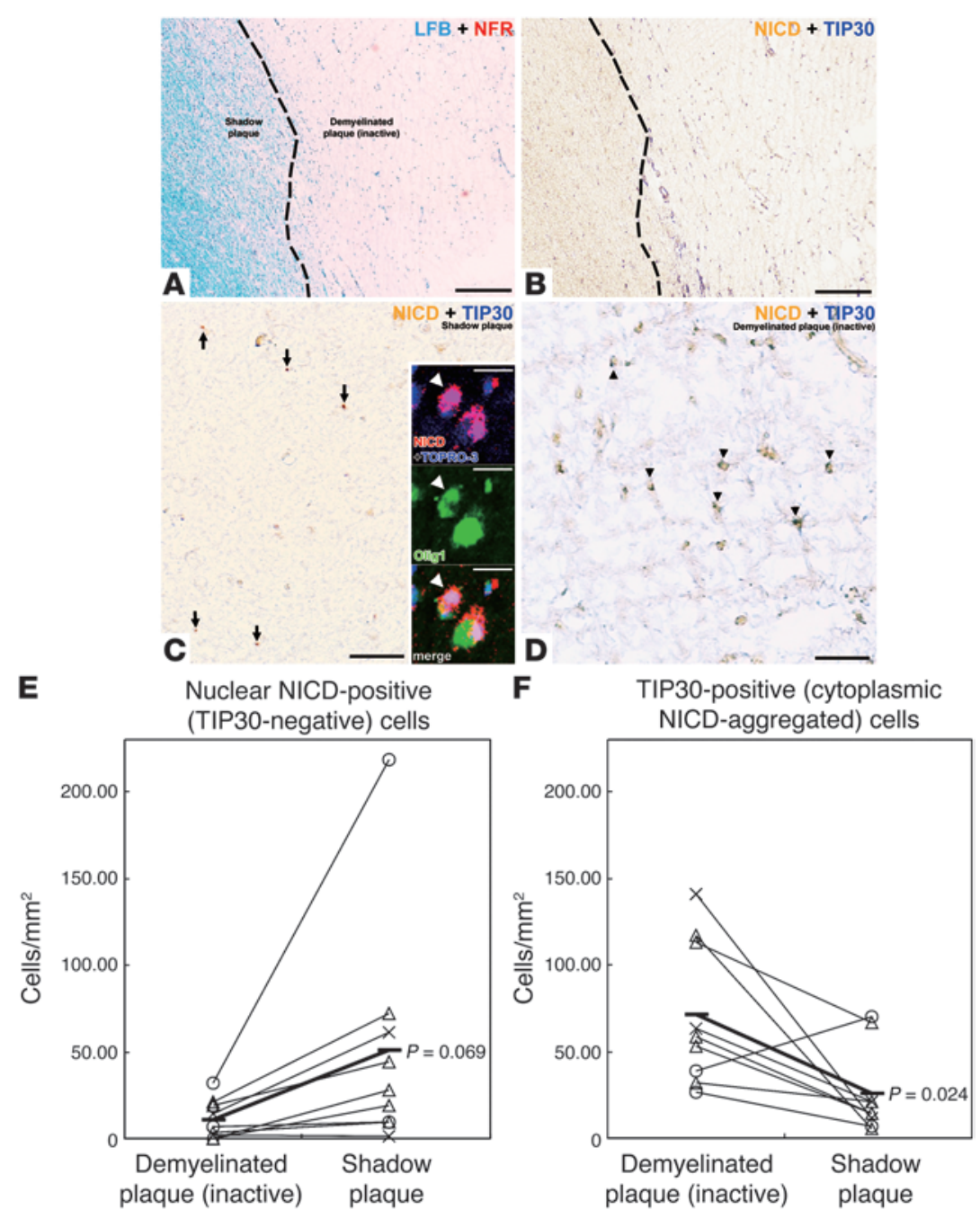

\section{Figure 5}

TIP30-positive cells are substantially increased in chronically demyelinated plaques. (A and $\mathbf{B}$ ) Two consecutive sections were chosen for each MS case, and 1 was stained with LFB plus NFR to identify chronically demyelinated plaques and remyelinating shadow plaques (demarcated by broken line), while the other was double-labeled against NICD and TIP30 (occipital lobe cerebral lesion from 51-year-old female with secondary progressive MS is shown). (C) A photograph at higher magnification from a remyelinating shadow plaque in B showing nuclear NICD-positive cells (arrows; the majority of these cells were positive for nuclear Olig1, an OPC marker [ref. 24] as shown in inset). (D) A photograph at higher magnification from a chronically demyelinated plaque in B, showing cytoplasmic TIP30 (cytoplasmic NICD-aggregated) cells (arrowheads). (E and F) Graphs showing results of statistical analysis for the densities of nuclear NICD-positive cells and cytoplasmic TIP30-positive (cytoplasmic NICDaggregated) cells, respectively. Data representing the same specimens are connected with lines. Bold lines indicate the average of results from 9 specimens. Circles indicate relapse-remitting MS, triangles indicate secondary-progressive MS, and X's indicate primary-progressive MS. The difference in the density of nuclear NICD-positive cells between chronically demyelinated plaques and remyelinating shadow plaques was not statistically significant $(P=0.069$; note that nuclear NICD is rapidly turned over and therefore difficult to detect; see main text), whereas a significant increase in the density of TIP30-positive cells was noted in chronically demyelinated plaques when compared with remyelinating shadow plaques $(P=0.024)$. Scale bars: $200 \mu \mathrm{m}(\mathbf{A}$ and $\mathbf{B}) ; 50 \mu \mathrm{m}$ (C and D); $20 \mu \mathrm{m}$ (insets in $\mathbf{C}$ ). exception of a moderate expression in cerebellum (33). Considering the similarity between the 2 diseases in respect to the remyelination failure, we thought that Lamin B1 might also be overexpressed in MS lesions and possibly associated with the NICD aggregates that include Nup153. Consistent with previous reports $(33,34)$, expression of Lamin B1 was virtually undetectable in control cerebral white matter (Figure 4A), whereas expression was increased in MS demyelinated lesions (Figure 4B). Moreover, the subcellular localization of Lamin B1 was indeed associated with the NICD aggregates in the OPCs (Figure 4, C-E). In support of the results, Abs raised against MS plaques have been shown to detect Lamin B (35).

OPCs express TIP30, an Importin- $\beta$ inhibitor, in MS lesions. Among the molecules we found in the cytoplasmic aggregates (NICD, ref. 15; Nup153, ref. 36; and Lamin B1, ref. 37), all possess NLS and are thus targets of Importin $\beta$-mediated, NLS-dependent nuclear translocalization (16). Therefore, the observed pathology in MS may reflect a general impairment in Importin $\beta$-mediated nucleocytoplasmic transport in OPCs, not only blocking the axoglial Contactin/Notch1 signaling pathway within the cells (12) but also inhibiting the proper transportation of Nup153 and Lamin $\mathrm{B} 1$ into the nuclear envelope, resulting in the aggregates.

TIP30 (otherwise known as CC3) is a normal cellular tumor suppressor and proapoptotic protein induced by heat shock (38), irra- diation (39), aging (40), or TGF- $\beta 1$ (41). Defects in TIP30 are associated with metastasis of small cell lung carcinoma (42) and breast carcinoma (43) or with carcinogenesis of hepatocellular carcinoma (44). The baseline expression of TIP30 varies from organ to organ and is relatively low in brains and neural-derived cell lines (42). The crystal structure of TIP30 reveals an Importin $\beta$-binding domain (18); when expressed in excess, TIP30 does indeed form a complex with Importin $\beta$ and inhibits Importin $\beta$-mediated nuclear import, consequently increasing the sensitivity to death signals (19).

In agreement with a previous study (42), TIP30 was virtually absent in control subjects except for small vessels (Figure 4F), whereas the expression was markedly upregulated in MS demyelinated lesions (Figure 4G). Subcellular localization of TIP30 was associated with that of Importin $\beta$ in the OPCs (Figure $4, \mathrm{H}-\mathrm{J})$. In a few cells in selected cases of MS, relatively lower expressions of TIP30 were observed. In such cases, Importin $\beta$ not associated with TIP30 eventually entered the nucleus (Figure $4, \mathrm{~K}-\mathrm{M}$ ), consistent with the theory that TIP30 acts as an inhibitor for Importin $\beta$-mediated nucleocytoplasmic transport $(18,19)$. These results suggest that the upregulated TIP30 in MS lesions interferes with axoglial Contactin/Notch1 signaling in the OPCs via the inhibition of nuclear translocalization of NICD, resulting in failure of remyelination. 

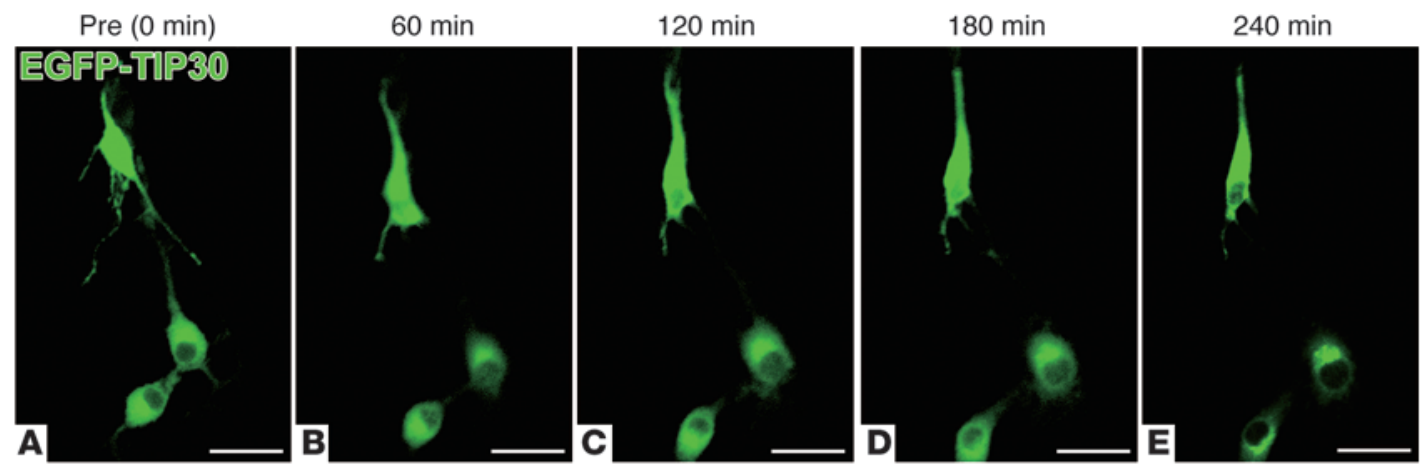

Pretreatment

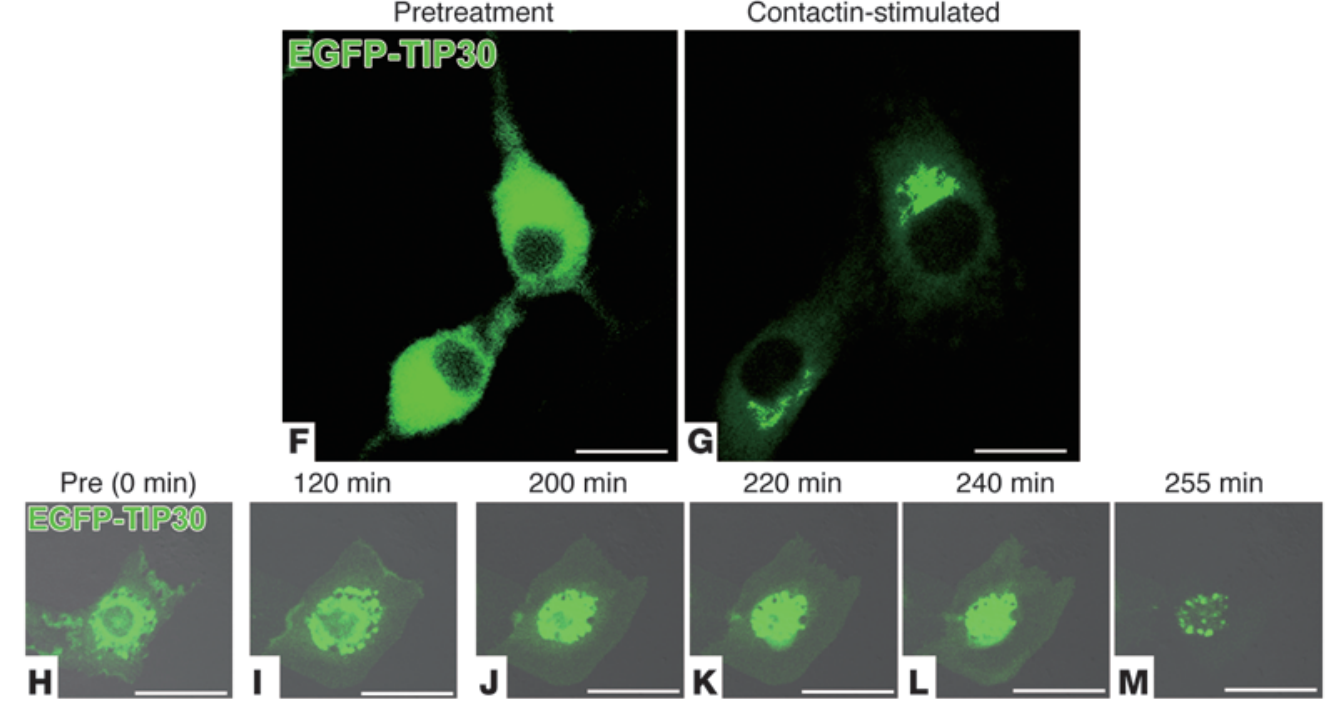

Contactin-stimulated

\section{Figure 6}

TIP30 forms cytoplasmic aggregates upon Contactin stimulation and increases susceptibility to cell death in vitro. (A-E) A series of captured pictures from a time-lapse recording of EGFP-TIP30-transfected OLN-93 cells upon Contactin stimulation in vitro. TIP30, initially expressed diffusely in the cytoplasm, becomes confined to perinuclear areas within a few hours after Contactin stimulation. The original recording is available as Supplemental Video 1. (F and G) Photographs at higher magnification of prestimulation (0 minutes) and post-Contactin stimulation (240 minutes) from $\mathbf{A}-\mathbf{E}$, showing perinuclear cytoplasmic aggregations of TIP30 upon Contactin stimulation. (H-M) In those cells with relatively high expressions of TIP30, perinuclear aggregations of TIP30 upon Contactin stimulation resulted in breakdown of the nuclear envelope and subsequent cell death (as seen in $\mathbf{M}$ ) within several hours. The original recording is available as Supplemental Video 2 (nontransgenic control is shown in Supplemental Video 3). Scale bars: $40 \mu \mathrm{m}$ (A-E); $20 \mu \mathrm{m}(\mathbf{F}-\mathbf{M})$.

TIP30-positive cells are less frequent in remyelinating shadow plaques. Among 10 MS samples included in the present study, 9 contained remyelinating shadow plaques, as determined by the presence of abnormally thin and short myelin and also immature oligodendrocytes (20). In the remyelinating shadow plaques, nuclear NICDpositive cells were detected, and most of them were positive for nuclear Olig1, suggestive of OPCs (Figure 5C). To further clarify the involvement of TIP30 in remyelination failure, we performed statistical analysis for the density of TIP30-positive/cytoplasmic NICD-aggregated cells and TIP30-negative/nuclear NICD-positive cells and compared chronically demyelinated plaques (without sign of ongoing demyelination; known as "inactive" lesions) and remyelinating shadow plaques (Figure 5, A-D).

The results (Figure 5, E and F) revealed that the density of TIP30negative/nuclear NICD-positive cells has a tendency to be higher in remyelinating shadow plaques than in chronically demyelinated plaques, although the difference is not statistically significant $\left(51.34 \pm 67.16\right.$ cells $/ \mathrm{mm}^{2}$ in remyelinating shadow plaques and $10.95 \pm 11.12$ cells $/ \mathrm{mm}^{2}$ in chronically demyelinated plaques; aver- age $\pm \mathrm{SD}, P=0.069$ ). This may be attributable in part to the difficulty in detecting nuclear NICD due to its rapid turnover (26). The density of TIP30-positive/cytoplasmic NICD-aggregated cells, which may reflect the degree of nucleocytoplasmic transport failure, is significantly greater in chronically demyelinated plaques and not as great in remyelinating shadow plaques $\left(71.40 \pm 41.65 \mathrm{cells} / \mathrm{mm}^{2}\right.$ in chronically demyelinated plaques and $24.76 \pm 24.92 \mathrm{cells} / \mathrm{mm}^{2}$ in remyelinating shadow plaques; average $\pm \mathrm{SD}, P=0.024)$. These results suggest that upregulated TIP30 is associated with remyelination failure in MS.

TIP30 blocks the nuclear translocalization of NICD and differentiation. The cell line OLN-93 resembles OPCs or immature oligodendrocytes in morphological and antigenic properties (45) and has been previously used to demonstrate the nuclear translocalization of NICD upon Contactin stimulation (12). In addition, OLN-93 cells were positive for nuclear Olig1 (not shown), suggesting that this cell line may be relevant to OPCs recruited for remyelination (24) or the preserved OPCs in MS lesions. In order to confirm that TIP30 acts as an inhibitor of Contactin/Notch1 signaling 


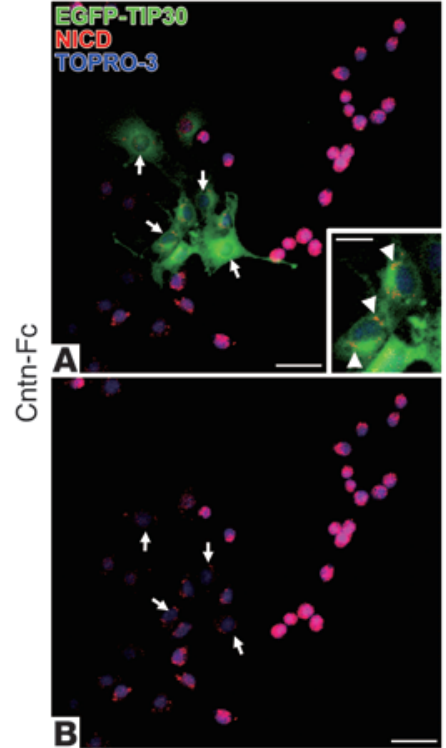

D

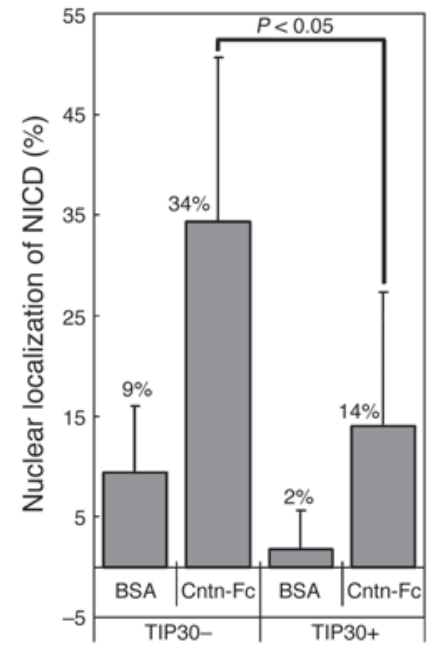

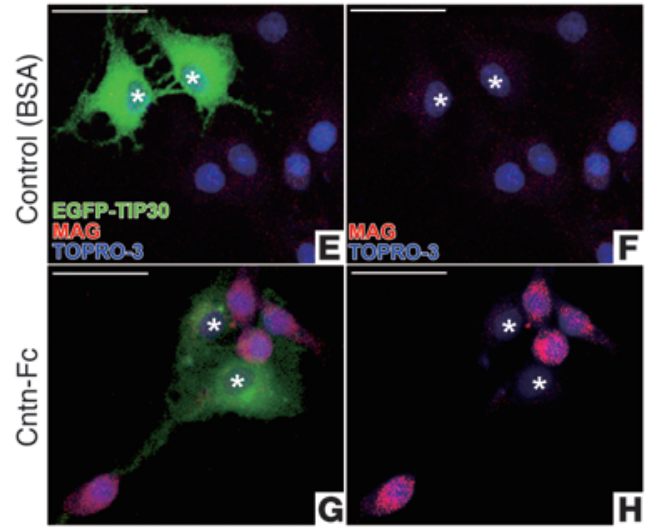

C
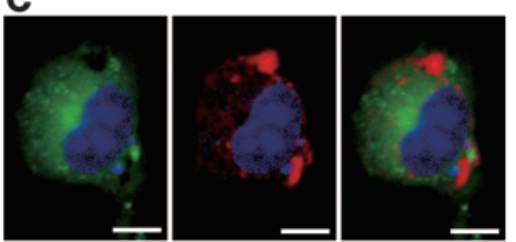

EGFP-

PDI

Merge

TIP30

TOPRO-3

Figure 7

Inhibition of nuclear translocalization of NICD and myelin protein expressions by TIP30 in vitro. (A and B) When OLN-93 cells are induced to differentiate via Contactin stimulation for 29 hours, nuclear translocalization of NICD occurs in naive cells but not in TIP30-positive cells (arrows). NICD in such cells are trapped in cytoplasm (arrowheads in inset). (C) TIP30 is expressed in the cytoplasm, outside the ER (the region positive for PDI), similarly to OPCs in MS lesions. (D) Nuclear translocation ratio of NICD is reduced about $60 \%$ (from $34 \%$ to $14 \%$ ) by TIP30. Note that the result is likely to be an underestimate because of cell death induced by TIP30 overexpression, as shown in Figure 6. (E-H) Contactin stimulation, in contrast to BSA control, promotes the expression of MAG in naive OLN-93 cells (12) but not in TIP30-positive cells (asterisks). Similar results were obtained for CNPase (Supplemental Figure 7). Note that pictures shown are 1D-rendered images from Z stack series (confocal slice image is shown in Figure 6). Error bars in D denote SD. Scale bars: $50 \mu \mathrm{m}$ (A and B); $20 \mu \mathrm{m}$ (inset in A); $10 \mu \mathrm{m}$ (C); $50 \mu \mathrm{m}$ (E-H).

and causes differentiation arrest of the OPCs, leading to remyelination failure, we transfected and EGFP tagged TIP30 (44) into OLN-93 cells and induced their differentiation with soluble Contactin-Fc as has been previously described (12). Time-lapse recordings of intracellular distributions of TIP30 in OLN-93 cells revealed that TIP30 is initially distributed diffusely in cytoplasm but upon Contactin-Fc stimulation becomes confined to perinuclear areas and forms granule-like aggregates morphologically similar to those observed in MS lesions (Figure 6, A-G, and Supplemental Video 1).

In those cells with overwhelming expression, TIP30 formed perinuclear aggregates and induced breakdown of nuclear membranes, resulting in cell death within a few hours (Figure 6, H-M, and Supplemental Video 2; nontransgenic cells are shown in Supplemental Video 3), supporting the view that TIP30 increases susceptibility to apoptotic cell death (19).

Even though only cells with relatively low expression of TIP30 were available for further analysis due to the aforementioned cell death, nuclear translocalization of NICD upon Contactin-Fc stimulation was clearly inhibited in TIP30-positive cells and NICD was trapped in the cytoplasm (Figure 7, A and B). The subcellular localization of TIP30 was outside the ER (as identified by PDI), similar to the OPCs in MS lesions (Figure 7C). Statistical analysis showed an approximately $60 \%$ reduction in the NICD nuclear translocalization ratio upon Contactin stimulation in cells with TIP30 expression, although the result is likely to be underestimated due to cell death (Figure 7D).

Contactin/Notch1 signaling has been shown to induce expression of myelin-associated glycoprotein (MAG) (12), a marker of mature oligodendrocytes, and the expression of MAG is indeed induced in these cells but not in those positive for TIP30 (Figure 7, E-H). Similar results were also observed for the expression of CNPase (Supplemental Figure 7). Possibly because of the short duration of TIP30 expression and Contactin stimulation in vitro (hours) in comparison with the OPCs in MS lesions (years), the NICD aggregates were less prominent in the cultured cells than in the OPCs in MS lesions and immunocytochemical detections of other NLS-tagged proteins were unremarkable (data not shown). 


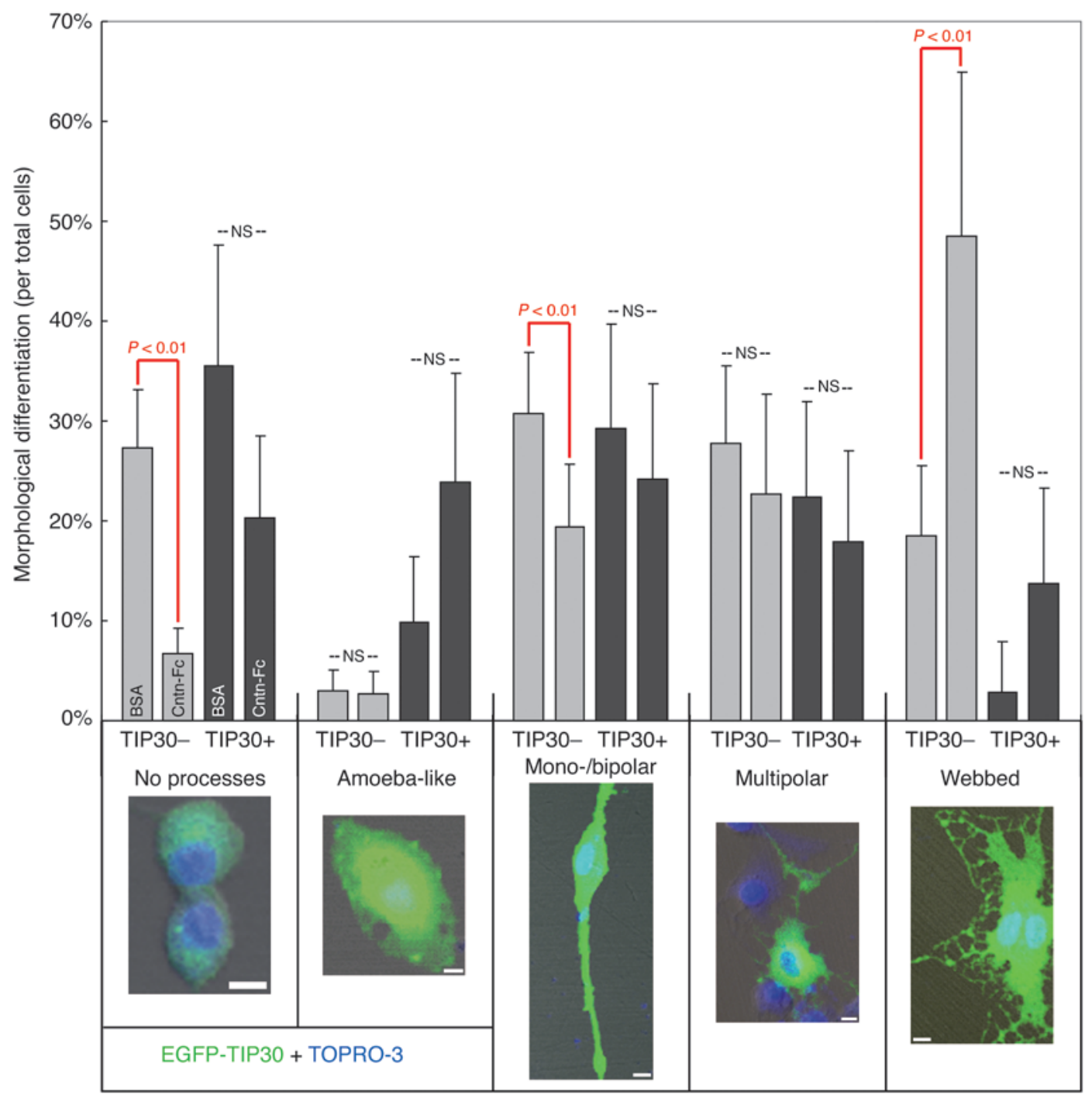

Figure 8

TIP30 inhibits morphological differentiation of OPC cells in vitro. When naive OLN-93 cells are induced to differentiate via Contactin stimulation for 29 hours, morphological differentiation (an increase in the percentage of differentiated webbed cells $[P=0.001]$ and decreases in the percentages of cell without processes $[P<0.001]$ and mono-/bipolar cells $[P=0.005]$ ) was observed in comparison with BSA control. These changes were not observed (statistically not significant; $P>0.05$ ) in TIP30-positive OLN-93 cells, suggesting TIP30 inhibits the morphological differentiation of OPC cells. Morphology of the cells was categorized into 5 groups (see Methods), and their typical appearances are shown. Error bars denote SD. Scale bars: $10 \mu \mathrm{m}$.

Differentiation of typically bipolar OPCs into myelinating oligodendrocytes involves remarkable morphological differentiation. Contactin/Notch 1 signaling induced by Contactin-Fc in naive OLN-93 cells indeed resulted in the reduction of cells without processes or mono-/bipolar cells, while cells with well-developed (webbed) processes were significantly increased (Figure 8). In TIP30-positive cells, however, such morphological differentiation was not observed (Figure 8). These results together suggest that TIP30 inhibits the differentiation of OPCs into oligodendrocytes both biochemically and morphologically.

\section{Discussion}

We have shown that many, if not all, chronically demyelinated axons in MS lesions express Contactin on their surfaces; ordinar- ily, this protein would instruct the preserved OPCs to differentiate into myelinating cells (12). Notch1-positive OPCs are increased in the Contactin-positive demyelinated lesions, and the receptor is indeed activated and sequentially cleaved to NICD. However, nuclear translocalization of NICD, which is necessary for the differentiation process, is blocked, and both the differentiation of OPCs into myelinating cells and remyelination are consequently inhibited. NICD is arrested and aggregated in cytoplasm, along with nuclear transporter Importin $\beta$ and NPC protein Nup153. In addition, ectopically overexpressed Lamin B1, a protein whose genetic duplication causes ADLD (33), was observed in the aggregates. Each of the proteins observed in the aggregates (NICD, ref. 15; Nup153, ref. 36; and Lamin B1, ref. 37) contain NLS and thus are targets of Importin $\beta$-mediated nuclear transport (16). TIP30, 


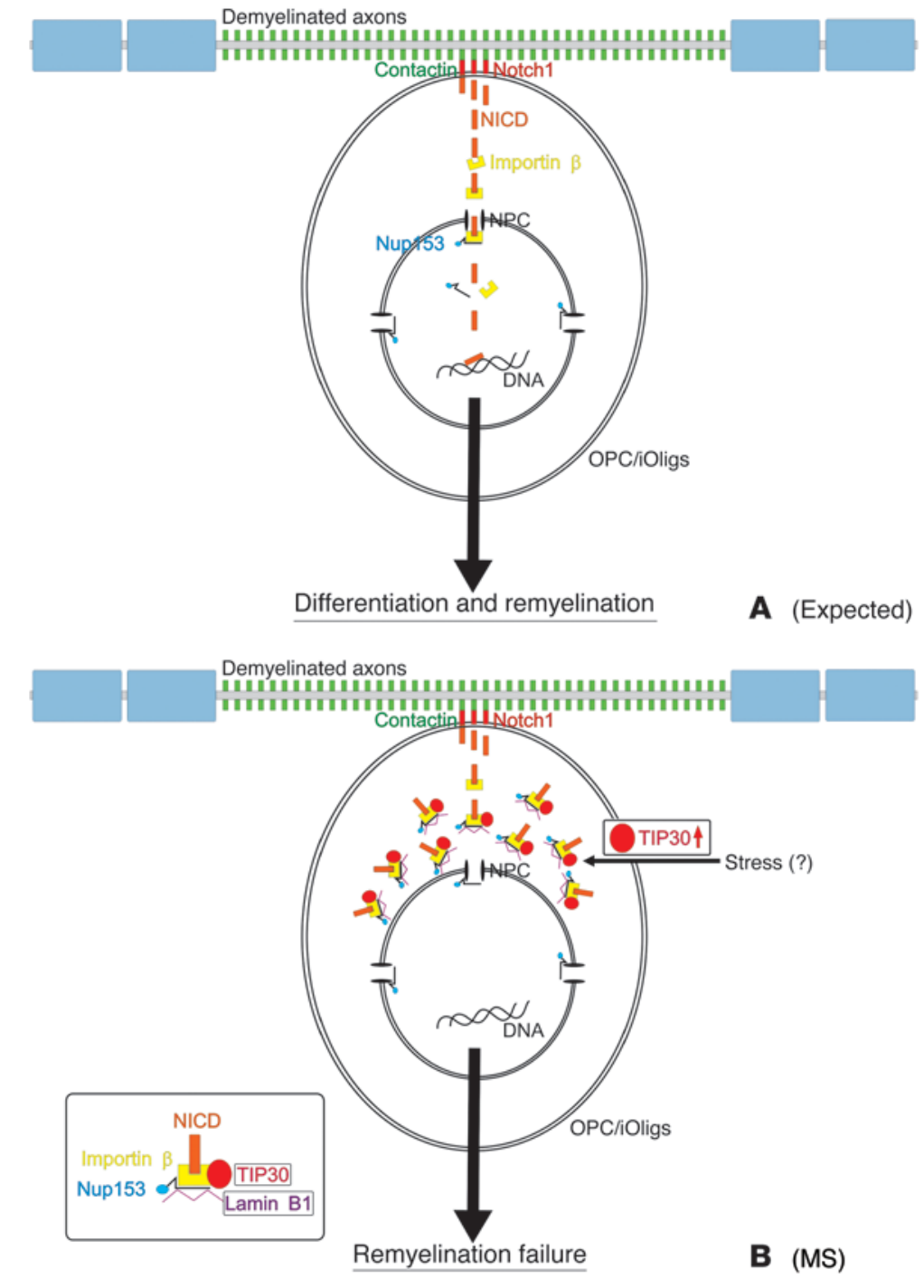

\section{Figure 9}

TIP30 inhibits oligodendroglial nucleocytoplasmic transport and arrests remyelination in MS. (A) A schematic drawing to show what is theoretically expected during remyelination when the preserved OPCs are naive. Oligodendroglial Notch1 is cleaved to NICD upon its ligation with axonal Contactin. NICD is then translocated into nucleus by nuclear transporter Importin $\beta$ in an NLS-dependent manner, promoting the expression of myelin-related genes and thereby inducing the maturation of oligodendrocytes for remyelination. (B) Conclusion of the present study of chronically demyelinated plaques in MS. Even though NICD is formed in the preserved OPCs, its nuclear translocalization is inhibited by TIP30, which directly blocks Importin $\beta$, thereby causing remyelination failure. TIP30 inhibits both the expression of myelin proteins and morphological differentiation. The abnormal expression of TIP30 may be mediated by extracellular stresses (see Discussion). iOligs, immature oligodendrocytes. a tumor suppressor and proapoptotic protein inhibiting Importin $\beta$-mediated nuclear transport (19) is abnormally upregulated and associated with the NICD aggregates. Overexpression of TIP30 in OPC cells in vitro blocked the Contactin/Notch 1 signaling by inhibiting nuclear translocalization of NICD and consequently prevented the maturation of OPC cells into oligodendrocytes. Together, our observations suggest that the preserved OPCs in MS lesions - in which nucleocytoplasmic transport is impaired by abnormal expressions of TIP30 - are not naive but pathogenic (schematically summarized in Figure 9).

While we analyzed MS brains using the axoglial Contactin/ Notch1 signaling (12) as a scaffold, our observations showing Nup153 and Lamin B1 in the cytoplasmic aggregates suggest that the nucleocytoplasmic transport mediated by Importin $\beta$ in general may have failed in the preserved OPCs in MS lesions. In this view, it is important to note that remyelination occurs after Cuprizone-induced chemical demyelination in OPC-targeted Notch1ablated transgenic mice (22), suggesting that additional pathways other than those involving Notch 1 exist and may also contribute to remyelination. Elucidation of such additional pathways and possible involvement of Importin $\beta$ therein awaits further study.
Our findings have several implications for the pathophysiology of MS and future therapeutic strategies. First, even though axonal pathologies are reported in MS $(4,14)$, many demyelinated axons are capable of expressing Contactin to induce myelinogenesis; thus, the remyelination failure may not be entirely due to lack of axonal signals. Second, our results indicate that the preserved OPCs in MS are not naive but pathogenic and that their nucleocytoplasmic transport is impaired due to abnormally upregulated TIP30. Hence, approaches, including ours $(20,46)$ or others' $(10,47)$ to promote remyelination solely by inducing the differentiation of endogenous OPCs into myelinating cells in MS may require amendment. To the extent that TIP30 is induced by specific environmental factors in MS lesions, the same holds true for approaches that would utilize exogenously derived OPCs. Systemic blockade of TIP30 may not be straightforward because of the potential for tumorigenesis (44). It is therefore essential to clarify the factor or factors inducing the abnormal upregulation of TIP30 in MS. TIP30 has been shown to be induced by stresses such as heat shock (38), irradiation (39), and aging (40) as well as by TGF- $\beta 1$ (41).

In addition, we must be cautious when using EAE animals in the study of remyelination, since nucleocytoplasmic transport of 
NICD seems intact in this model (48). Our preliminary observations also indicated that TIP30 is not induced in the demyelinated lesions observed in animals with myelin oligodendrocyte glycoprotein (amino acids 1-125) EAE (Jin Nakahara, unpublished observations) and thus do not reflect the findings in the present study. Generation of an animal model in which TIP30 is overexpressed in OPCs in vivo may be technically challenging, as our observations showed that such cells are highly susceptible to cell death. Primary nonimmune-mediated apoptosis of oligodendrocytes is observed in newly formed MS lesions (49), and possible influences of TIP30 in this phenomenon should be further studied.

Finally, our observations showing overexpression of Lamin B1, an NLS-tagged protein, in the OPCs raise the possibility that similar pathophysiologies underlie both MS and ADLD. Very recently, similar cytoplasmic aggregations of Importin $\beta$ in proportion to disease progression, suggestive of nucleocytoplasmic transport failure, have been reported in anterior horn cells of the mutant $\mathrm{Cu} / \mathrm{Zn}$-superoxide dismutase (G93A) transgenic mouse model of amyotrophic lateral sclerosis (50). Abnormal cytoplasmic accumulations of nuclear transport protein have also been observed in hippocampal neurons of brain samples from Alzheimer disease patients (51). Nucleocytoplasmic transport and its failure may thus be a novel paradigm underlying a wide spectrum of neural diseases, including MS.

\section{Methods}

Human brain specimens. All of the experimental procedures were carried out in accordance with institutional guidelines at Keio University, and the analysis of human specimens was approved by the institutional review board at the Human Brain and Spinal Fluid Resource Center (Los Angeles, California, USA), from which all human specimens used in this study were obtained. The human brain specimens used in this study were described in our previous study (20). The cerebra of 10 deceased MS patients and 5 control patients without brain diseases were investigated in this study. Each MS specimen contained at least 1 demyelinated plaque. Autolysis time varied from 11 to 58 hours (average: 21 hours) for MS patients and from 9 to 19 hours (average: 12 hours) for control patients. Available clinical history about the patients and lesion characteristics are described in our previous report (20). Fresh-frozen specimens were thawed and fixed in 4\% PFA-PBS (Wako) at $4^{\circ} \mathrm{C}$ for 20 hours before proceeding to sectioning.

Cryostat sectioning. Fixed specimens were washed thoroughly in PBS (Ambion; Applied Biosystems) and immersed in a PBS solution containing $20 \%$ sucrose at $4{ }^{\circ} \mathrm{C}$ until the specimens sank. The cryoprotected specimens were frozen and embedded in a compound medium (Tissue-Tek OCT Compound; Sakura) and then sliced into $10-\mu \mathrm{m}$ thick sections using a cryostat microtome (CM3050; Leica) at $-25^{\circ} \mathrm{C}$. Slices were collected on glass slides with an aminosilane precoat (Matsunami Glass Ind. Ltd.) and stored at $-80^{\circ} \mathrm{C}$ until use.

Myelin staining. A luxol fast blue (LFB) (Acros Organics) solution $(0.1 \%$ LFB in $95 \%$ ethanol containing $0.05 \%$ acetic acid, stained overnight at $60^{\circ} \mathrm{C}$ ) and a nuclear fast red (NFR) counterstain (stained for 5 minutes at room temperature; Vector Laboratories) were routinely used for myelin staining to distinguish demyelinated lesions. Lithium carbonate solution (0.05\%; Wako) was used for color differentiation of the LFB. Slides were viewed and photos were taken using a stereotactic optical microscope equipped with a CCD camera (COOLSCOPE; Nikon).

Immunohistochemistry. Antigen retrieval was performed by placing the slides in boiled 0.01 M citrate buffer solution (Muto Pure Chemicals) for 10 minutes. After the procedure, slides were incubated with primary Abs diluted in $0.5 \%$ skim milk (BD) in PBS for 2 days at $4^{\circ} \mathrm{C}$ and then with secondary Abs diluted in $0.5 \%$ skim milk in PBS overnight at $4{ }^{\circ} \mathrm{C}$. The slides were thoroughly washed in PBS in between each Ab hybridization. The specimens were mounted in VECTASHIELD (Vector Laboratories) and viewed under a confocal laser scanning microscope (Digital Eclipse C1; Nikon). Double-labeling experiments using 2 rabbit Abs (i.e., antiNICD and anti-TIP30, as shown in Figure 5) were performed as described previously (20). In brief, quenching of internal peroxidase activity was performed by $3 \%$ hydrogen peroxidase ( 10 minutes at room temperature; Wako) and the Abs targeting the nuclei (i.e., anti-NICD) were visualized first by immunoperoxidase staining with 3,3-diaminobenzidine, tetrahydrochloride (DAB) (brown; Dojindo). Slides were then washed once in running water for 10 minutes and twice in $0.1 \mathrm{M}$ glycine- $\mathrm{HCl}$ (Wako) at $\mathrm{pH} 2.2$ for 30 minutes to remove the bound Abs. The second set of Abs (i.e., antiTIP30) was administered similarly and visualized by immunoperoxidase staining with 4-chloro-1-naphthol (blue; Wako). Slides were viewed and photos were taken using a stereotactic optical microscope equipped with a CCD camera (COOLSCOPE; Nikon).

Lesion staging and statistical analysis. For the statistical analysis shown in Figure 5, chronically demyelinated plaques (inactive) were determined by the complete loss of LFB staining and lack of myelin-phagocytosing macrophages (52), and remyelinating shadow plaques were determined as previously described (20). One out of $10 \mathrm{MS}$ samples analyzed included nearby actively demyelinating lesions, as identified by the presence of macrophages phagocytosing LFB-positive particles, and this case was omitted from the following statistical analysis as a result of the difficulty in precisely identifying the remyelinating shadow plaques. Two consecutive slices were selected for each MS case. One slide was then stained with LFB plus NFR, whereas the other was double-labeled for NICD and TIP30. Using the LFB plus NFR slide, the locations of chronically demyelinated plaques (inactive) and remyelinating shadow plaques were stereotactically mapped by COOLSCOPE (Nikon). Photographs of immunostained slides (double-labeled for NICD and TIP30) were stereotactically taken for corresponding chronically demyelinated plaques and remyelinating shadow plaques in accordance with the results from the LFB plus NFR staining. Digital photographs representing $0.146 \mathrm{~mm}^{2}$ were taken with a CCD camera at a resolution of $1280 \times 960$ pixels with an original magnification of $\times 200$ under COOLSCOPE. At least 3 different photographs of areas that were more than $1 \mathrm{~mm}$ apart from each other in $x$ and $y$ directions were randomly taken for every chronically demyelinated plaque and remyelinating shadow plaque. The number of TIP30-negative/nuclear NICD-positive cells and TIP30-positive/cytoplasmic NICD-aggregated cells in each photograph was counted. The average density of the cells per $\mathrm{mm}^{2}$ was calculated for each MS sample (for both cell types) as graphed in Figure 5, E and F. The statistical significance of the differences in average density between chronically demyelinated plaques and remyelinating shadow plaques was determined by 2 -sided, paired Student's $t$ test, with $P<0.05$ considered significant.

Cell culture, transfection, and Contactin stimulation. OPC cell line OLN-93 (45) (a generous gift from Zhi-Cheng Xiao, Singapore General Hospital, Singapore; ref. 12) was cultured in DMEM (Wako) containing $10 \%$ heatinactivated FBS (GIBCO, Invitrogen), $100 \mathrm{U} / \mathrm{ml}$ penicillin, and $100 \mu \mathrm{g} / \mathrm{ml}$ streptomycin (Sigma-Aldrich) and incubated at $37^{\circ} \mathrm{C}$ in humidified $5 \%$ $\mathrm{CO}_{2}$. After a passage with $0.2 \%$ Trypsin (BD Biosciences) in PBS, the cells were cultured in 6 -well culture plates $(35-\mathrm{mm}$ diameter per well; BD Biosciences) for 1 day to ensure their attachment to the plates. Culture medium was changed to serum- and antibiotic-free DMEM with $1.8 \mu \mathrm{g} / \mathrm{ml}$ Metafectene Pro (Biontex) and $0.6 \mu \mathrm{g} / \mathrm{ml}$ pEGFP-C2/TIP30 plasmid (a generous gift from Hua Xiao and Isamu Hoshino, Michigan State University, East Lansing, Michigan, USA; ref. 44) and incubated for 14 hours to achieve transfection. The transfected cells were further cultured in fresh serum- and antibiotic-free DMEM for 10 hours, and then stimulation of 
the cells was accomplished by changing the medium to serum-free DMEM with $100 \mathrm{U} / \mathrm{ml}$ penicillin, $100 \mu \mathrm{g} / \mathrm{ml}$ streptomycin, and either $0.5 \mu \mathrm{g} / \mathrm{ml}$ Contactin-Fc (AbCys) or $0.5 \mu \mathrm{g} / \mathrm{ml} \mathrm{BSA} \mathrm{(Invitrogen)} \mathrm{as} \mathrm{a} \mathrm{control,} \mathrm{then}$ incubating for 29 hours before proceeding to immunocytochemistry.

Time-lapse recording. At the time of Contactin-Fc stimulation, a 6-well culture plate was placed on a confocal laser-scanning microscope equipped with a Thermo Plate (temperature set at $37^{\circ} \mathrm{C}$; Tokai Hit) and time-lapse recording software (Nikon). DMEM containing $6 \mathrm{mg} / \mathrm{ml} \mathrm{HEPES} \mathrm{was} \mathrm{used}$ to avoid $\mathrm{pH}$ changes, and no remarkable changes in color as indicated by phenol red were observed during our relatively short recording time (up to 4.5 hours). Time-lapse recordings of EGFP (TIP30) fluorescence were performed at 5-minute intervals as shown in Supplemental Videos 1-3.

Immunocytochemistry and statistical analysis. Cultured cells were fixed with 4\% PFA-PBS for 20 minutes at room temperature, antigen retrieved with boiled $0.01 \mathrm{M}$ citrate buffer solution for 10 minutes, permeabilized with $0.1 \%$ Triton X-100 (Sigma-Aldrich) in PBS for 5 minutes at room temperature, and then blocked with $1 \%$ FBS in PBS for 20 minutes at room temperature before $\mathrm{Ab}$ administration. Abs were diluted in $1 \% \mathrm{FBS}$ in PBS, and primary Abs were incubated for 12 hours at $37^{\circ} \mathrm{C}$ and then with secondary Abs for 1 hour at $37^{\circ} \mathrm{C}$, with thorough washes with $\mathrm{PBS}$ in between. The stained cells were viewed with a confocal laser-scanning microscope. For the statistical analysis of NICD nuclear-translocalization ratio (Figure 7D), pictures were randomly taken from both Contactin-stimulated and BSA-stimulated (control) groups, and all of the cells in every picture were classified by the localization of NICD (nucleus or cytoplasm) and by the expression of TIP30 (TIP30-positive or -negative). A total of 112 TIP30-positive cells and 489 TIP30-negative cells were classified. The statistical difference was analyzed by 2 -sided Student's $t$ test; differences of $P<0.05$ were considered significant. For the statistical analysis of morphological differentiation (Figure 8), cells were classified by their morphology (i.e., cells without processes, amoeba-like cells [hypertrophic cells without remarkable processes], mono- or bipolar cells, multipolar cells, and webbed cells; typical examples are shown in Figure 8). At least 3 independent experiments were performed, and a total of 130 TIP30-positive cells and 1723 TIP30-negative cells were classified. The statistical difference was analyzed by 2 -sided Student's $t$ test, with $P<0.05$ considered significant.

$A b s$. The primary Abs used in this study included anti-Contactin mouse monoclonal Abs (clone 41; BD Biosciences), anti-neurofilament rabbit polyclonal Abs (AbD Serotec), anti-Notch1 rabbit polyclonal Abs (Abcam), anti-Val ${ }_{1744}$-cleaved NICD rabbit polyclonal Abs (Cell Signaling Technology), anti-CNPase mouse monoclonal Abs (clone 11-5B; Sigma-Aldrich),
anti-Olig1 mouse monoclonal Abs (clone 257219; R\&D Systems), antiHes5 rabbit polyclonal Abs (Chemicon; Millipore), anti-Importin $\beta$ mouse monoclonal Abs (clone 31H4; Sigma-Aldrich), anti-Nup153 mouse monoclonal Abs (clone QE5; Abcam), anti-PDI mouse monoclonal Abs (clone 1D3; Abcam), anti-Lamin B1 mouse monoclonal Abs (clone 119D5-F1; Chemicon; Millipore), anti-TIP30 rabbit polyclonal Abs (Abcam), antiGFAP rabbit polyclonal Abs (Dako), and anti-MAG rabbit polyclonal Abs (53). For the control experiments (Supplemental Figure 2), mouse and rabbit IgG (Jackson ImmunoResearch Laboratories Inc.) were substituted for primary Abs. The secondary Abs and reagents used in this study included indocarbocyanine-conjugated (Cy3-conjugated) or FITC-conjugated antirabbit IgG goat polyclonal Abs (Jackson ImmunoResearch Laboratories Inc.), Cy3- or FITC-conjugated anti-mouse IgG goat polyclonal Abs (Jackson ImmunoResearch Laboratories Inc.), and TO-PRO-3 iodide nuclear stain (Molecular Probes; Invitrogen).

\section{Acknowledgments}

Jin Nakahara was supported by the Japan Society for the Promotion of Science as a research fellow and by the Keio University KANRINMARU Project as an assistant professor. This work was supported by Grants-in-Aid and Special Coordination Funds for Promoting Science and Technology from the Ministry of Education, Culture, Sports, Science and Technology of Japan. We would like to acknowledge the patients, their families, and staff contributing to the Human Brain and Spinal Fluid Resource Center (Los Angeles, California, USA), which is sponsored by the National Institute of Neurological Disorders and Stroke/National Institute of Mental Health, the National Multiple Sclerosis Society, the VA Greater Los Angeles Healthcare System, and the Veterans Health Services and Research Administration (Department of Veterans Affairs). We are grateful to Zhi-Cheng Xiao (Singapore General Hospital) and Hua Xiao and Isamu Hoshino (Michigan State University) for providing experimental reagents and Hiroaki Asou (Keio University) for essential discussions.

Received for publication February 25, 2008, and accepted in revised form October 15, 2008.

Address correspondence to: Jin Nakahara, Department of Neurology, Keio University School of Medicine, 35 Shinanomachi, Shinjuku-ku, Tokyo 160-8582, Japan. Phone: 81-3-3353-1211 ext. 63573; Fax: 81-3-5360-1524; E-mail: nakahara@sc.itc.keio.ac.jp.
1. Patrikios, P., et al. 2006. Remyelination is extensive in a subset of multiple sclerosis patients. Brain. 129:3165-3172.

2. Patani, R., Balaratnam, M., Vora, A., and Reynolds, A. 2007. Remyelination can be extensive in multiple sclerosis despite a long disease course. Neuropathol. Appl. Neurobiol. 33:277-287.

3. Wolswijk, G. 1998. Chronic stage multiple sclerosis lesions contain a relatively quiescent population of oligodendrocyte precursor cells. J. Neurosci. 18:601-609.

4. Chang, A., Tourtellotte, W.W., Rudick, R., and Trapp, B.D. 2002. Premyelinating oligodendrocytes in chronic lesions of multiple sclerosis. N. Engl. J. Med. 346:165-173.

5. Franklin, R.J. 2002. Why does remyelination fail in multiple sclerosis? Nat. Rev. Neurosci. 3:705-714.

6. John, G.R., et al. 2002. Multiple sclerosis: re-expression of a developmental pathway that restricts oligodendrocyte maturation. Nat. Med. 8:1115-1121.

7. Back, S.A., et al. 2005. Hyaluronan accumulates in demyelinated lesions and inhibits oligodendrocyte progenitor maturation. Nat. Med. 11:966-972.

8. Charles, P., et al. 2002. Re-expression of PSA-NCAM by demyelinated axons: an inhibitor of remyelination in multiple sclerosis? Brain. 125:1972-1979.

9. Satoh, J., Tabunoki, H., Yamamura, T., Arima, K., and Konno, H. 2007. TROY and LINGO-1 expression in astrocytes and macrophages/microglia in multiple sclerosis lesions. Neuropathol. Appl. Neurobiol. 33:99-107.

10. Mi, S., et al. 2005. LINGO-1 negatively regulates myelination by oligodendrocytes. Nat. Neurosci. 8:745-751.

11. Kotter, M.R., Li, W.W., Zhao, C., and Franklin, R.J. 2006. Myelin impairs CNS remyelination by inhibiting oligodendrocyte precursor cell differentiation. J. Neurosci. 26:328-332.

12. Hu, Q.D., et al. 2003. F3/contactin acts as a functional ligand for notch during oligodendrocyte maturation. Cell. 115:163-175.

13. Pierre, K., Dupouy, B., Allard, M., Poulain, D.A., and Theodosis, D.T. 2001. Mobilization of the cell adhesion glycoprotein F3/contactin to axonal surfaces is activity dependent. Eur. J. Neurosci. 14:645-656.

14. Trapp, B.D., et al. 1998. Axonal transection in the lesions of multiple sclerosis. N. Engl. J. Med. 338:278-285.

15. Kopan, R., Nye, J.S., and Weintraub, H. 1994. The intracellular domain of mouse Notch: a constitutively activated repressor of myogenesis directed at the basic helix-loop-helix region of MyoD. Development. 120:2385-2396

16. Fried, H., and Kutay, U. 2003. Nucleocytoplasmic transport: taking an inventory. Cell. Mol. Life Sci. 60:1659-1688.

17. Görlich, D., Vogel, F., Mills, A.D., Hartmann, E., and Laskey R.A. 1995. Distinct functions for the two importin subunits in nuclear protein import. Nature. 377:246-248.

18. El Omari, K., Bird, L.E., Nichols, C.E., Ren, J., and Stammers, D.K. 2005. Crystal structure of CC3 (TIP30): implications for its role as a tumor suppressor. J. Biol. Chem. 280:18229-18236.

19. King, F.W., and Shtivelman, E. 2004. Inhibition of nuclear import by the proapoptotic protein CC3. 
Mol. Cell. Biol. 24:7091-7101.

20. Nakahara, J., and Aiso, S. 2006. Fc receptor-positive cells in remyelinating multiple sclerosis lesions. J. Neuropathol. Exp. Neurol. 65:582-591.

21. Durbec, P., Gennarini, G., Goridis, C., and Rougon, G. 1992. A soluble form of the F3 neuronal cell adhesion molecule promotes neurite outgrowth. J. Cell Biol. 117:877-887.

22. Stidworthy, M.F., et al. 2004. Notch1 and Jagged 1 are expressed after CNS demyelination, but are not a major rate-determining factor during remyelination. Brain. 127:1928-1941.

23. Kondo, T., and Raff, M. 2000. Basic helix-loop-helix proteins and the timing of oligodendrocyte differentiation. Development. 127:2989-2998.

24. Arnett, H.A., et al. 2004. bHLH transcription factor Olig1 is required to repair demyelinated lesions in the CNS. Science. 306:2111-2115.

25. Setoguchi, T., and Kondo, T. 2004. Nuclear export of Olig2 in neural stem cells is essential for ciliary neurotrophic factor-induced astrocyte differentiation. J. Cell Biol. 166:963-968.

26. Hansson, E.M., et al. 2006. Recording Notch signaling in real time. Dev. Neurosci. 28:118-127.

27. Yang, W., and Musser, S.M. 2006. Nuclear import time and transport efficiency depend on importin $\beta$ concentration. J. Cell Biol. 174:951-961.

28. Lock, C., et al. 2002. Gene-microarray analysis of multiple sclerosis lesions yields new targets validated in autoimmune encephalomyelitis. Nat. Med. 8:500-508.

29. Walther, T.C., et al. 2001. The nucleoporin Nup153 is required for nuclear pore basket formation, nuclear pore complex anchoring and import of a subset of nuclear proteins. EMBO J. 20:5703-5714.

30. Gustin, K.E., and Sarnow, P. 2001. Effects of poliovirus infection on nucleo-cytoplasmic trafficking and nuclear pore complex composition. EMBO J. 20:240-249.

31. Daigle, N., et al. 2001. Nuclear pore complexes form immobile networks and have a very low turnover in live mammalian cells. J. Cell Biol. 154:71-84.

32. Smythe, C., Jenkins, H.E., and Hutchison, C.J. 2000.
Incorporation of the nuclear pore basket protein Nup153 into nuclear pore structures is dependent upon lamina assembly: evidence from cell-free extracts of Xenopus eggs. EMBO J. 19:3918-3931.

33. Padiath, Q.S., et al. 2006. Lamin B1 duplications cause autosomal dominant leukodystrophy. Nat. Genet. 38:1114-1123.

34. Lin, F., and Worman, H.J. 1997. Expression of nuclear Lamins in human tissues and cancer cell lines and transcription from the promoters of the Lamin A/C and B1 genes. Exp. Cell Res. 236:378-384.

35. García, D.M., Weigum, S.E., and Koke, J.R. 2003. GFAP and nuclear lamins share an epitope recognized by monoclonal antibody J1-31. Brain Res. 976:9-21.

36. Nakielny, S., Shaikh, S., Burke, B., and Dreyfuss, G. 1999. Nup153 is an M9-containing mobile nucleoporin with a novel Ran-binding domain. EMBO J. 18:1982-1995.

37. Mical, T.I., and Monteiro, M.J. 1998. The role of sequences unique to nuclear intermediate filaments in the targeting and assembly of human lamin B: evidence for lack of interaction of lamin B with its putative receptor. J. Cell Sci. 111:3471-3485.

38. Narita, N., et al. 2002. Analysis of heat-shock related gene expression in head-and-neck cancer using cDNA arrays. Int. J. Radiat. Oncol. Biol. Phys. 53:190-196.

39. Chaudhry, M.A., Chodosh, L.A., McKenna, W.G., and Muschel, R.J. 2003. Gene expression profile of human cells irradiated in G1 and G2 phases of cell cycle. Cancer Lett. 195:221-233.

40. Lesur, I., and Campbell, J.L. 2004. The transcriptome of prematurely aging yeast cells is similar to that of telomerase-deficient cells. Mol. Biol. Cell. 15:1297-1312.

41. Lindfors, K., Halttunen, T., Kainulainen, H., and Mäki, M. 2001. Differentially expressed CC3/TIP30 and rab11 along in vivo and in vitro intestinal epithelial cell crypt-villus axis. Life Sci. 69:1363-1372.

42. Shtivelman, E. 1997. A link between metastasis and resistance to apoptosis of variant small cell lung carcinoma. Oncogene. 14:2167-2173.

43. Zhao, J., et al. 2007. TIP30/CC3 expression in breast carcinoma: relation to metastasis, clinicopathologic parameters, and P53 expression. Hum. Pathol. 38:293-298.

44. Ito, M., et al. 2003. TIP30 deficiency increases susceptibility to tumorigenesis. Cancer Res. 63:8763-8767.

45. Richter-Landsberg, C., and Heinrich, M. 1996. OLN-93: a new permanent oligodendroglia cell line derived from primary rat brain glial cultures. J. Neurosci. Res. 45:161-173.

46. Nakahara, J., et al. 2003. Signaling via immunoglobulin Fc receptors induces oligodendrocyte precursor cell differentiation. Dev. Cell. 4:841-852.

47. Mi, S., et al. 2007. LINGO-1 antagonist promotes spinal cord remyelination and axonal integrity in MOG-induced experimental autoimmune encephalomyelitis. Nat. Med. 13:1228-1233.

48. Seifert, T., Bauer, J., Weissert, R., Fazekas, F., and Storch, M.K. 2007. Notch1 and its ligand Jagged 1 are present in remyelination in a T-cell- and antibodymediated model of inflammatory demyelination. Acta Neuropathol. 113:195-203.

49. Barnett, M.H., and Prineas, J.W. 2004. Relapsing and remitting multiple sclerosis: pathology of the newly forming lesion. Ann. Neurol. 55:458-468.

50. Zhang, J., et al. 2006. Altered distributions of nucleocytoplasmic transport-related proteins in the spinal cord of a mouse model of amyotrophic lateral sclerosis. Acta Neuropathol. 112:673-680.

51. Sheffield, L.G., Miskiewicz, H.B., Tannenbaum, L.B., and Mirra, S.S. 2006. Nuclar pore complex proteins in Alzheimer disease. J. Neuropathol. Exp. Neurol. 65:45-54.

52. van der Valk, P., and De Groot, C.J. 2000. Staging of multiple sclerosis (MS) lesions: pathology of the time frame of MS. Neuropathol. Appl. Neurobiol. 26:2-10.

53. Nakahara, J., et al. 2001. Myelin basic protein is necessary for the regulation of myelin-associated glycoprotein expression in mouse oligodendroglia. Neurosci. Lett. 298:163-166. 